\title{
On Operator Inequalities due to Ando-Kittaneh-Kosaki
}

By

\author{
Jun Ichi FuJII* and Masatoshi FuJII*
}

\begin{abstract}
Operator norm inequalities due to Ando-Kittaneh-Kosaki for positive operators $A, B$ and a non-negative operator monotone function $f$ on $[0, \infty)$ are discussed: Main inequality is $\|f(A)-f(B)\| \leqq\|f(|A-B|)\|$. It is shown that the equality holds for invertible $A, B$ and non-linear $f$ if and only if $A=B$ and $f(0)=0$. Similarly, from the Kittaneh-Kosaki inequality, we show that $\|f(A)-f(B)\|=f^{\prime}(t)\|A-B\|$ for $A, B \geqq t>0$ and nonlinear $f$ if and only if $A=B$.
\end{abstract}

\section{$\S$ 1. Introduction}

From the viewpoint of the Schatten $p$-norm, Kittaneh and Kosaki [2] showed some inequalities for the operator norm. Recently, T. Ando [1] showed two comparison theorems for unitarily invariant norms of positive semi-definite matrices making use of Ky Fan norm technique, and summed up the interesting inequalities related to operator monotone functions.

A real function $f$ is called operator monotone (on $[0, \infty)$ ) if $A \leqq B$ implies $f(A) \leqq f(B)$ for (bounded linear) positive operators $A, B$ on a Hilbert space. In the below, we assume an operator monotone function is non-negative. Then, a main inequality of the Ando-Kittaneh-Kosaki is as follows:

$$
\|f(A)-f(B)\| \leqq\|f(|A-B|)\| \text {. }
$$

On the other hand, Kittaneh and Kosaki discussed an equation:

(b) For $A, B \geqq t>0,2 t\|A-B\|=\left\|A^{2}-B^{2}\right\|$ if and only if $A=B$.

Note that (b) is the equality case for $f(t)=t^{1 / 2}$ in the following inequality by them:

$$
\|f(A)-f(B)\| \leqq f^{\prime}(t)\|A-B\| \quad \text { for } \quad A, B \geqq t>0 .
$$

Communicated by H. Araki, October 29, 1987.

* Department of Mathematics, Osaka Kyoiku University, Osaka 543, Japan. 
In this note, by examining such inequalities, we shall consider the equality conditions for non-linear $f$. It is shown that equality in (a) holds for invertible $A, B$ if and only if $A=B$. The condition $A=B$ is also equivalent to the equality in the Kittaneh-Kosaki inequality (c). In addition, as an application of the inequality (a), we shall give an improvement of [2; Theorem 3.4]:

$$
\|\log (A+t)-\log (B+t)\|<\log (2\|A-B\| / t) \quad \text { for } \quad 0<t<\|A-B\| .
$$

\section{§ 2. Ando-Kittaneh-Kosaki Inequalities}

First, we shall consider the equality condition

$$
\|f(A)-f(B)\|=\|f(|A-B|)\|
$$

for the inequality (a) which is stated in [1; Theorem 1], [2; Theorem 2.3]. Since the equation (d) always holds for linear $f$, we assume that $f$ is non-linear. Then, it is natural to expect that (d) implies $A=B$. But, even in a commutative case, a counter-example is given: The equality (d) holds for $f(t)=t^{1 / 2}, A=\left(\begin{array}{ll}1 & 0 \\ 0 & 0\end{array}\right)$ and $B=\left(\begin{array}{ll}2 & 0 \\ 0 & 1\end{array}\right)$. In this example, it should be noted that $A$ is not invertible. As a matter of fact, we have the following:

Theorem 1. If $f$ is a non-linear non-negative operator monotone function on $[0, \infty)$, then $\|f(A)-f(B)\|=\|f(|A-B|)\|$ for positive invertible operators $A$ and $B$ if and only if $A=B$ and $f(0)=0$.

Proof. Since a non-linear operator monotone function is strictly concave, for any $t>0$ and positive invertible $X$ there is $\varepsilon=\varepsilon(t, X)>0$ such that $f(t+X)-f(X)+\varepsilon \leqq f(t)-f(0)$, hence

$$
\|f(t+X)-f(X)\|<f(t)-f(0) .
$$

Therefore, if either $\|A-B\|>0$ or $f(0)>0$, then

$$
\begin{aligned}
& a=\|f(\|A-B\|+A)-f(A)\|<f(\|A-B\|), \text { and } \\
& b=\|f(\|A-B\|+B)-f(B)\|<f(\|A-B\|) .
\end{aligned}
$$

Putting $c=\max \{a, b\}$, we have

$$
f(A)-f(B)=f(A-B+B)-f(B) \leqq f(\|A-B\|+B)-f(B) \leqq c,
$$

and similarly $f(B)-f(A) \leqq c$. Since $|f(A)-f(B)| \leqq c$, it follows that

$$
\|f(A)-f(B)\| \leqq c<f(\|A-B\|)=\|f(|A-B|)\| .
$$


Thus the equality shows that $\|A-B\|=0$ and $f(0)=0$. The converse is clear.

Now, we apply Theorem 1 and the inequality (a) to typical operator monotone functions. The following inequalities are due to Ando [1]:

Corollary 1.1. The following inequalities hold for positive operators $A$ and $B$, and the equality for invertible $A, B$ holds only when $A=B$ :

(i) ||$A^{p}-B^{p}|| \leqq \||A-B|^{p}||$ for $0<p<1$, and

(ii) $\|\log (A+1)-\log (B+1)\| \leqq\|\log (|A-B|+1)\|$.

The inequality (ii) in the above leads us an improvement of [2; Theorem 3.4]. From the viewpoint of this note, the following inequality shows that the equality condition itself is not reasonable in their theorem.

Corollary 1.2. For positive operators $A, B$ with $0<t<\|A-B\|$ for some constant $t,\|\log (A+t)-\log (B+t)\|<\log (2\|A-B\| / t)$.

Proof. Since $\|C-D\|>1$ for $C=A / t$ and $D=B / t$, Corollary 1.1. (ii) implies that

$$
\begin{aligned}
\|\log (A+t)-\log (B+t)\| & =\|\log (C+1)-\log (D+1)\| \\
& \leqq\|\log (|C-D|+1)\|=\log (\|C-D\|+1) \\
& <\log (2\|C-D\|)=\log (2\|A-B\| / t) .
\end{aligned}
$$

\section{§3. Inverse Inequalities}

Symmetrically, we shall discuss an inverse inequality for the inverse function of operator monotone one, cf. [1; Theorem 3]:

Corollary 1.3. If a continuous increasing unbounded function $g$ on $[0, \infty)$ with $g(0)=0$ has the inverse function $f$ which is operator monotone, then $\|g(A)-g(B)\| \geqq\|g(|A-B|)\|$ for positive operators $A$ and $B$. Moreover, the equality for invertible $A, B$ holds for nonlinear $g$ if and only if $A=B$.

Proof. Applying the inequality (a) for $g(A)$ and $g(B)$, we have

$$
f(\|g(A)-g(B)\|) \geqq\|f(g(A))-f(g(B))\|=\|A-B\| .
$$

It follows from monotonity of $g$ that

$$
\|g(A)-g(B)\|=g(f(\|g(A)-g(B)\|)) \geqq g(\| A-B||)=\|g(|A-B|)\| .
$$

The second statement follows from Theorem 1.

Like Corollary 1.1, we can get the operator norm version of [1; Corollary 4] 
(cf. [1; Lemma 5]):

Corollary 1.4. The following inequalities hold for positive operators $A$ and $B$, and the equality for invertible $A, B$ holds only when $A=B$ :

(i) $\left\|e^{A}-e^{B}\right\| \geqq\left\|e^{|A-B|}-1\right\|$,

(ii) $\left\|A^{P}-B^{P}\right\| \geqq|||A-B|^{P} \|$ for $p \geqq 1$, and

(iii) ||$A^{p} \log (A+1)-B^{p} \log (B+1)|| \geqq|||A-B|^{p} \log (|A-B|+1)||$ for $p \geqq 1$.

\section{§ 4. Estimation by Derivative}

As a generalization of the van Hemmen-Ando theorem [3; Proposition 4.1], Kittaneh and Kosaki established the following inequality [2; Theorem 3.1]: Let $f$ be a non-negative continuous operator monotone function on $[0, \infty)$, and $A, B$ positive operators with $0 \leqq a \leqq A, 0 \leqq b \leqq B$. Then, for every operator $X,\|f(A) X-X f(B)\| \leqq C(a, b)\|A X-X B\|$ where $C(a, b)=f^{\prime}(a)$ when $a=b$, $=(f(a)-f(b)) /(a-b)$ otherwise. In this section, we shall consider the equality condition in (c), that is, the case $X=1$ and $a=b$ in the above. We note that (b) is a special case of this: Let $f(t)=t^{1 / 2}$. Since $A^{2}, B^{2} \geqq c^{2}$, we have that $\|A-B\| \leqq$ $(2 t)^{-1}\left\|A^{2}-B^{2}\right\|$ by (c). In this case, the equality is eqivalent to $A=B$. More generally:

Theorem 2. Let $f$ be a non-negative non-linear operator monotone function on $(0, \infty)$, and $A, B$ positive operators with $A, B \geqq c>0$ for some scalar $c$. Then, $\|f(A)-f(B)\|=f^{\prime}(c)\|A-B\|$ if and only if $A=B$.

Proof. Suppose $\|f(A)-f(B)\|=f^{\prime}(c)\|A-B\|$. Here we use the integral representation of $f: f(x)=\alpha+\beta x+\int_{0}^{\infty}(t: x) \operatorname{dm}(t)$ where $t: x$ means the parallel sum $t x /(t+x), \alpha=f(0), \beta=\lim _{t \rightarrow \infty} f(t) / t$ and $d \mu(t)=\{t /(1+t)\} d m(t)$ is a positive Radon measure. Notice that the support of $m$ is non-trivial since $f$ is non-affine.

Putting $X=(t+A)^{-1}(A-B)(t+B)^{-1}=t^{-2}(t: A-t: B)$, we have

$$
\begin{aligned}
\|f(A)-f(B)\| & =\left\|\beta(A-B)+\int(t: A-t: B) d m(t)\right\| \\
& \leqq \beta\|A-B\|+\int t^{2}\|X\| d m(t) \\
& \leqq \beta\|A-B\|+\int t^{2}\left\|(t+A)^{-1}\right\|\|A-B\|\left\|(t+B)^{-1}\right\| d m(t) \\
& \leqq\left\{\beta+\int t^{2}(t+a)^{-1}(t+b)^{-1} d m(t)\right\}\|A-B\|
\end{aligned}
$$




$$
\begin{aligned}
& \leqq\left\{\beta+\int t^{2}(t+c)^{-2} d m(t)\right\}\|A-B\| \\
& =f^{\prime}(c)\|A-B\|=\|f(A)-f(B)\|,
\end{aligned}
$$

where $a=\min \sigma(A)$ and $b=\min \sigma(B)$. Therefore, on the support of $m$, we have two equations up to null sets:

$$
\begin{array}{cl}
\|X\|=\left\|(t+A)^{-1}\right\|\|A-B\|\left\|(t+B)^{-1}\right\| & \text { by }(*), \\
(t+a)^{-1}(t+b)^{-1}\|A-B\|=(t+c)^{-2}\|A-B\| & \text { by (**). }
\end{array}
$$

Here suppose $A \neq B$ to the contrary. Then (2) implies $a=b=c$. We may assume that there exists a state $\omega$ with $\omega(X)=\omega(|X|)=\|X\|$ since the condition is symmetric for $A, B$ and $X=X^{*}$. Noting that $\omega(|Y Z|) \leqq\|Y\| \omega(|Z|)$, it follows from (1) that the following:

$$
\begin{aligned}
\|X\|=\omega(|X|) & \leqq\left\|(t+A)^{-1}\right\|\|A-B\| \omega\left((t+B)^{-1}\right) \\
& \leqq\left\|(t+A)^{-1}\right\|\|A-B\|\left\|(t+B)^{-1}\right\|,
\end{aligned}
$$

imply $\omega\left((t+B)^{-1}\right)=\left\|(t+B)^{-1}\right\|=1 /(t+b)$. Since $t: B=t\left(1-t(t+B)^{-1}\right)$, we have $\omega(t: B)=t: b$. Similarly, since

$$
\begin{aligned}
\|X\|=\omega(|X|) & \leqq\left\|(t+B)^{-1}\right\|\|A-B\| \omega\left((t+A)^{-1}\right) \\
& \leqq\left\|(t+B)^{-1}\right\|\|A-B\|\left\|(t+A)^{-1}\right\|
\end{aligned}
$$

by the self-adjointness of $X$, we have $\omega(t: A)=t: a$. Therefore,

$$
t^{2} \omega(X)=\omega(t: A-t: B)=\omega(t: A)-\omega(t: B)=t: a-t: b=0,
$$

which implies $X=0$, hence $A=B$. This is a contradiction, that is, the equality implies $A=B$. The converse is clear.

Remark. It is essential that $f^{\prime}(c)$ dominates $C(a, b)$ and $f^{\prime}(t)$ on the spectra of $A$ and $B$. Indeed, the equation $\|f(A)-f(B)\|=C(a, b)\|A-B\|$ does not always imply $A=B$. For example, let $f(t)=t^{1 / 2}, A=1$ and $B=1 \oplus \varepsilon$ for $0<\varepsilon<1$. Then, $\|A-B\|=1-\varepsilon$ and $\|f(A)-f(B)\|=1-\varepsilon^{1 / 2}$. Since $C(a, b)=\left(1-\varepsilon^{1 / 2}\right) /(1-\varepsilon)$ for $a=1$ and $b=\varepsilon$, we have the equation $\|f(A)-f(B)\|=C(a, b)\|A-B\|$ although $A \neq B$.

\section{Acknowledgement}

The authors would like to express their thanks to the referee for his kind and warm suggestions. 


\section{References}

[1] Ando, T., Comparison of norms $\|f(A)-f(B)\|$ and $\|f(|A-B|)\|$, Preprint.

[2] Kittaneh, F. and Kosaki, H., Inequalities for the Schatten p-norm V, Publ. RIMS, Kyoto Univ., 23 (1986), 433-443.

[3] van Hemmen, J.L. and Ando, T., An inequality for trace ideals, Commun. Math. Phys., 76 (1980), 143-148. 